\title{
Using BIM for Improving Buildability in Small-Scale Construction Projects: A Comparative Case-Study
}

\author{
Fidel Puebla Canales ${ }^{1}$, Mauricio Loyola Vergara ${ }^{1}$ \\ ${ }^{1}$ Universidad de Chile, Chile \\ fidel.puebla@ug.uchile.cl \\ mloyola@uchile.cl
}

\begin{abstract}
Numerous authors have shown that using BIM has positive impacts on improving buildability levels on large-scale construction projects. This paper examines the impact of BIM on small-scale projects, particularly single-family homes. A series of case studies and interviews with architecture professionals were conducted. Results show that the benefits obtained are equivalent to those reported in the literature for larger projects, suggesting that the impact of BIM on improving buildability levels is similar for both small-scale and large-scale projects.
\end{abstract}

Keywords: BIM, Buildability, Small-Scale Projects

\section{INTRODUCCION}

La constructividad (buildability) se refiere al grado en el cual un determinado diseño permite una mayor facilidad y eficiencia de construcción, sujeto a todos los requerimientos del cliente y el proyecto (Loyola y Goldsack, 2010). En los proyectos arquitectónicos es un atributo especialmente importante pues diseños con mayor constructivdad facilitan el desarrollo de obras más eficientes, rápidas, seguras, económicas y de mejor calidad final.

La tecnología BIM (Building Information Modeling) ha sido sindicada como gran una impulsora de la mejora de constructividad en los proyectos debido a que los procesos de modelación, simulación y evaluación digital de los procesos constructivos permiten identificar prematuramente los problemas y corregirlos a tiempo. Distintos autores han reportado mejoras sustantivas de eficiencia en los procesos de diseño y construcción gracias a la utilización de esta tecnología. 


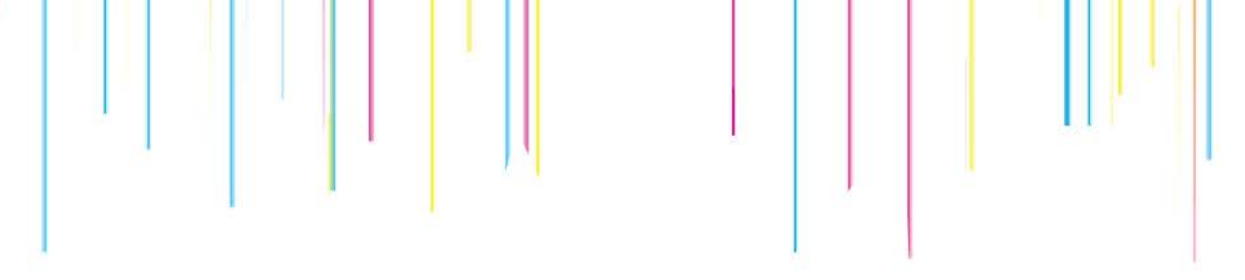

Sin embargo, la inmensa mayoría de los estudios están enfocados en edificios de gran escala y alta complejidad, tales como edificios hospitalarios, aeroportuarios, comerciales, de oficinas, culturales y otros similares. La principal razón esgrimida es que estos edificios plantean grandes desafíos constructivos, por lo que las mejoras de eficiencia obtenidas tienen considerables impactos en el costo y tiempo de los proyectos.

Por el contrario, el uso de BIM en proyectos de pequeña escala, tales como viviendas unifamiliares o edificios de equipamiento menor, está notablemente menos cubierto por la literatura. Es un vacío llamativo, pues es evidente que en estos proyectos también existen dificultades en sus procesos de construcción (aunque quizás diferentes a las encontradas en proyectos mayores). Naturalmente, surge la pregunta sobre cómo impacta el uso de BIM en la mejora de constructividad de los proyectos de pequeña escala.

Es una pregunta relevante, pues los requerimientos económicos y ambientales actuales exigen que todos los proyectos, sin excepción de escala, tengan procesos constructivos que hagan uso eficiente de los recursos.

\section{ANTECEDENTES}

\subsection{CONSTRUCTIVIDAD}

El concepto de constructividad fue originalmente propuesto por CIRIA (1983), desarrollado por Adams (1990) y posteriormente formalizado en un modelo de análisis propuesto por Loyola y Goldsack (2010). El modelo plantea 10 factores y 4 principios de constructividad. Los factores alertan sobre aquello de debe ser considerado en las decisiones de diseño y los principios orientan las decisiones de diseño, señalando la tendencia adecuada en la mayoría de las situaciones de diseño (Tabla 1). De acuerdo con el modelo, la correcta consideración de los factores y principios da origen a diseños que tienen mayor grado de constructividad. 


\subsection{BIM Y PEQUEÑA ESCALA}

Algunos autores han estudiado el uso de BIM en pequeña escala en general. Klaschka (2014) presentó una serie de casos de estudio muy detallados de pequeñas pequeñas y medianas empresas de Europa, mostrando cómo la tecnología y las metodologías de trabajo BIM pueden efectivamente adaptarse a oficinas y prácticas pequeñas y producir beneficios concretos en la calidad de los diseños y crecimiento de las oficinas. Por su parte, Bryde, Broquetas y Volm (2013) analizaron diferentes casos de proyectos internacionales y concluyeron que los beneficios obtenidos con BIM son muy superiores a las dificultades, destacando las mejoras en la coordinación, comunicación, costo, calidad y tiempo de obra entre las más importantes.

De forma similar, Alario (2014) sostuvo que el uso de BIM en proyectos de pequeña escala, aunque no permite aprovechar todo el potencial de la tecnología, sí ofrece interesantes beneficios que se traducen en una mejor optimización del trabajo, mejor calidad final y mejor servicio al cliente (Tabla 2).

Tabla 2: Beneficios y dificultades del uso de BIM en proyectos de pequeña escala. Fuente: elaboración propia en base a Alario (2014).

\begin{tabular}{|l|l|}
\hline \multicolumn{1}{|c|}{ Beneficios } & \multicolumn{1}{|c|}{ Dificultades } \\
\hline $\begin{array}{l}\text { Modelado rápido a nivel básico, con mucha } \\
\text { facilidad de realizar cambios y propuestas. }\end{array}$ & Coste de software. \\
\hline $\begin{array}{l}\text { Una vez modelado es muy rápido obtener } \\
\text { todas las vistas o secciones que necesitemos } \\
\text { sin ningún esfuerzo. }\end{array}$ & Necesidad de adaptación técnica. \\
\hline $\begin{array}{l}\text { Mostrar vistas 3D al cliente para que tome } \\
\text { decisiones, además de que se vende mejor un } \\
\text { proyecto en 3D. }\end{array}$ & Necesidad de formación. \\
\hline $\begin{array}{l}\text { Se puede crear alternativas dentro del modelo } \\
\text { para que el cliente las valore, no solo a nivel } \\
\text { visual, sino que sin ningún tipo de esfuerzo se } \\
\text { puede dar una valoración económica precisa } \\
\text { de cada una de las opciones, ofreciendo más } \\
\text { datos al cliente para escoger. }\end{array}$ & $\begin{array}{l}\text { Implicación de todos los agentes, para } \\
\text { que los técnicos los únicos que se adapten }\end{array}$ \\
\hline $\begin{array}{l}\text { Mediciones rápidas y adaptadas a la última } \\
\text { versión del modelo. }\end{array}$ & $\begin{array}{l}\text { Información sobre el uso que se le puede } \\
\text { dar. }\end{array}$ \\
\hline $\begin{array}{l}\text { Gestión del modelo por fases, como por ejem- } \\
\text { plo estado actual, demoliciones y reformado } \\
\text { sin tener que duplicar los planos. }\end{array}$ & $\begin{array}{l}\text { Un campo muy amplio, implicará especiali- } \\
\text { zación. }\end{array}$ \\
\hline $\begin{array}{l}\text { Obtención de cuadros de superficies sincroni- } \\
\text { zados con el documento de la memoria. }\end{array}$ & \\
\hline \begin{tabular}{l} 
Gestión de control de calidad" \\
\hline
\end{tabular} & \\
\hline
\end{tabular}




\section{METODOLOGIA}

La investigación consideró dos etapas. En la primera, se realizó una revisión de reportes sobre la mejora de constructividad gracias al uso de BIM en proyectos de mediana y gran escala, a fin de establecer una base comparativa para evaluar posteriormente el caso de los edificios de pequeña escala. Para el análisis se tomó como referencia el modelo de factores y principios (Tabla 1) y se puso especial énfasis en identificar los aspectos más relevantes determinantes de la eficiencia y facilidad de construcción.

La segunda etapa consistió en el análisis de proyectos de pequeña escala, (específicamente vivienda unifamiliar) desarrollados con BIM en Chile. Los resultados de este analisis son los que se presentan en este artículo.

Para la selección de la muestra de casos, se realizó un levantamiento de proyectos de vivienda desarrollados con BIM, a partir de información disponible en sitios web y publicaciones nacionales del área. Luego, se preseleccionaron todos aquellos casos en los que exístia suficiente información técnica disponible y en que los profesionales de proyecto manifestaron disponibilidad de participar en el estudio. Finalmente, para la muestra definitiva se eligieron 5 casos que cubrian los 3 tipos de uso de BIM descritos en 2.2., y además, que correspondieran a oficinas con diferentes características de tamaño, experiencia y alcance de sus servicios profesionales. Este último requisito derivó del análisis de literatura que alertó sobre el impacto de estas diferencias en el uso de BIM. Se establecieron 4 categorías:

- Que fuera un proyecto desarrollado por una oficina de arquitectura grande, con experiencia en el diseño de edificios de gran escala y complejidad con metodología BIM.

- Que fuera un proyecto desarrollado por una oficina de arquitectura pequeña, con experiencia en proyectos de remodelaciones, ampliaciones y habilitaciones.

- Que fuera un proyecto desarrollado por una oficina dedicada solo al diseño arquitectónico

- Que fuera un proyecto desarrollado por oficina dedicada tanto al diseño como a la construcción.

A continuación se indican los cinco casos estudiados, con sus arquitectos, ubicación, y año de construcción. Su categorización se muestra en la Figura 1.

- Casa Antilhue, Santiago. Badía y Soffia, 2016.

- Casa Lago Colico, Colico. Estudio Escabini, 2021.

- Casa Licán, Marina Puyehue. Arq Visión Arquitectos, 2021.

- Casa Piedra Blanca, Peñalolén. Lobos y Petrucelli, 2018

- Casa Las Colinas, Lo Barnechea. Luders, Gómez, Cruz y Labarca, 2020. 


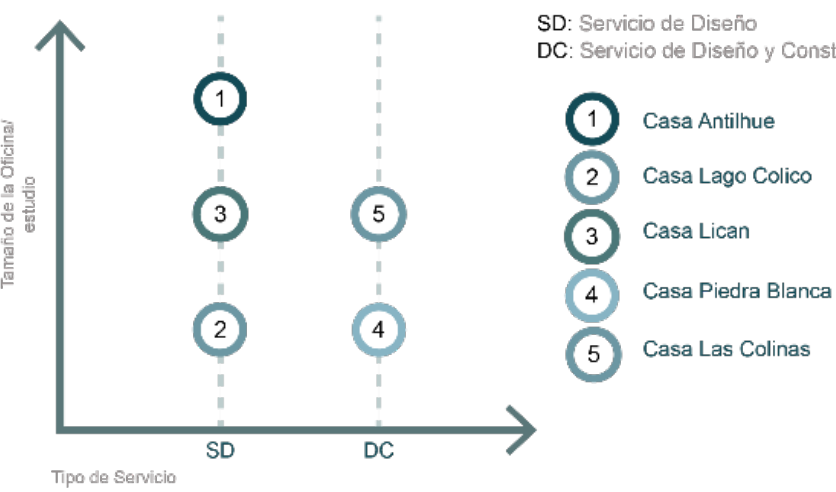

Figura 1: Categorización de los casos.
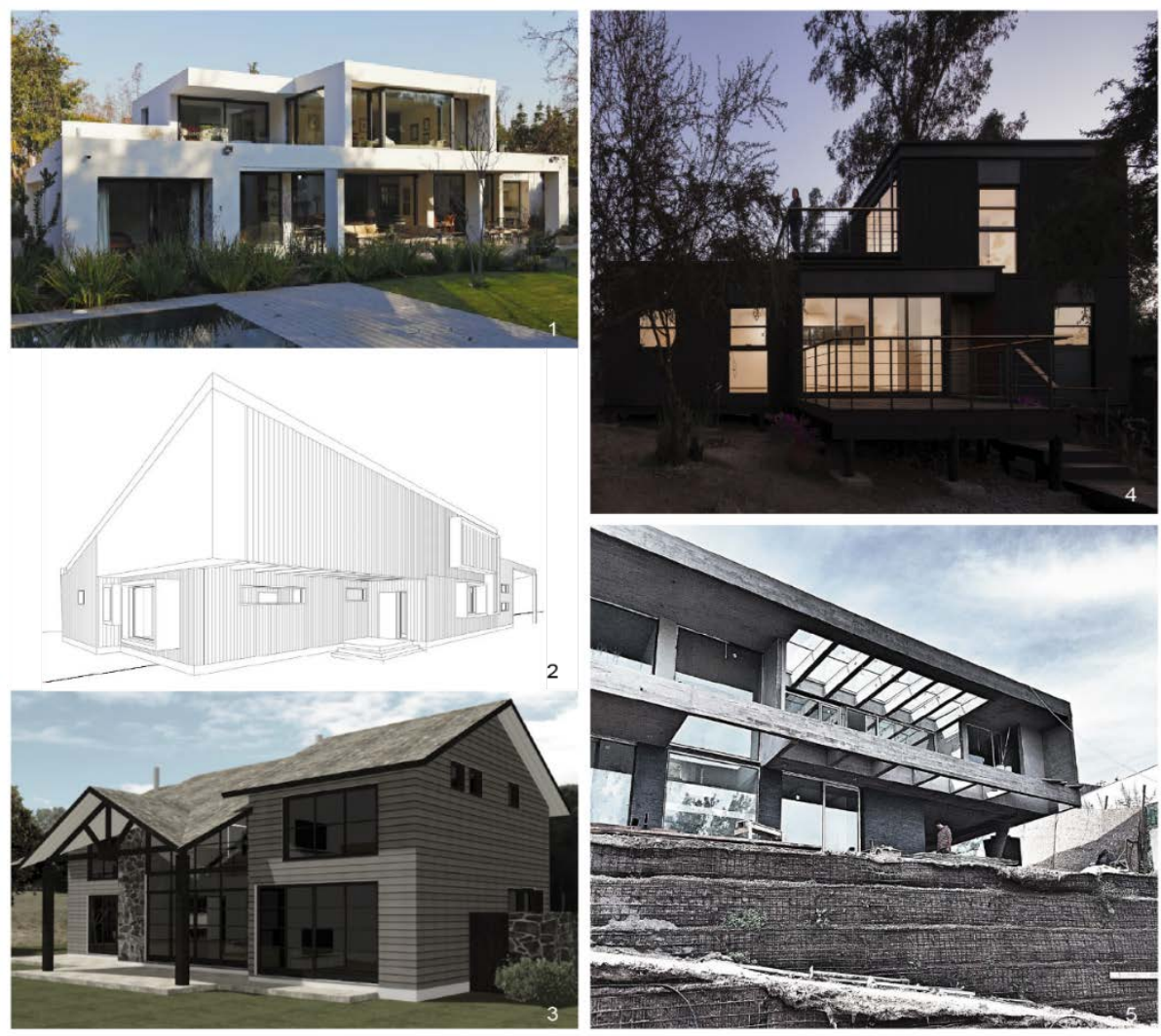

Figura 2. Casos de Estudio: 1) Casa Antilhue; 2) Casa Lago Colico; 3) Casa Licán; 4) Casa Piedra Blanca y 5) Casa Las Colinas. Fuente: 1) www.badia-soffia.cl/; 2) https://www.escabini.com/; 3) https://arqvision.cl/; 4) https://www. plataformaarquitectura.cl/ y 5) https://labarq.tumblr.com/. 
El análisis se realizó siguiendo una matriz adaptada de Camelio (2011). Primero, se realizó un análisis individual en profundidad de cada caso para identificar aspectos individuales relevantes. Luego, se realizó un análisis cruzado comparativo que permitió identificar replicaciones literales que validaran los hallazgos. Las fuentes de información fueron la documentación técnica disponible del proyecto y una entrevista semiestructurada a los profesionales encargados del diseño y/o construcción. El detalle de la documentación, cuestionario utilizado y transcripción de las entrevistas está disponible a investigadores que lo soliciten.

\section{RESULTADOS}

El resultado más claro y evidente, pero no sorpresivo, es que los proyectos que presentaron mayor consideración a los factores y principios de constructividad, y por lo tanto, mayor grado de constructividad general, fueron aquellos donde las tareas de diseño y construcción dependían de los profesionales de una misma oficina. Este resultado está ampliamente documentado en la literatura, y se explica, fundamentalmente, por la integración natural que ocurre en estas oficinas, por los incentivos explícitos existentes para alinear los objetivos de diseño y construcción y por el control completo que existe del proyecto.

En las oficinas donde estas tareas están separadas, el análisis muestra que el uso de BIM mejoró considerablemente la comunicación y coordinación entre profesionales. Estos factores están determinados por la claridad, cantidad, especificidad y calidad de la información; por la fluidez de la comunicación entre diseñadores y constructores, y por la coherencia, integración y complemento entre las distintas especialidades que intervienen. Este resultado es relevante, pues es perfectamente coincidente con lo reportado en proyectos de gran escala.

Los resultados también muestran que un factor de constructividad que se vio mejorado con el uso de BIM fue el relativo a materiales, es decir, la consideración de las características físicas, mecánicas y tecnológicas de los materiales, productos e insumos usados.

Con relación a los principios de constructividad, el análisis no arroja resultados concluyentes.

Según la categorización de gestión de información, sólo el proyecto Casa Antilhue logró un nivel BIM Integrado. Este modo de trabajo permitió que el proyecto tuviera un bajo nivel de errores y descordinaciones, lo que se tradujo en pocos conflictos de obra. Es importante destacar que este proyecto fue el que tenía la mayor superficie construida $\left(\sim 500 \mathrm{~m}^{2}\right.$, en el límite superior de la pequeña escala) y que fue realizado por una oficina que además desarrolla proyectos de gran envergadura. Según explicó el arquitecto responsable: "la metodología de trabajo es idéntica a la realizada en un proyecto grande (...) el edificio es menos complejo, pero el flujo de trabajo es igual". 
Los proyectos Casa Colico y Casa Piedra Blanca mostraron un método de trabajo de BIM no integrado unilateral. En ellos, las especialidades de terceros fueron modeladas por los propios profesionales de diseño a partir de información entregada en archivos DWG, lo que ciertamente disminuyó la eficiencia y obligó a priorizar tiempo de modelado. "El nivel de detalle [del modelo] cambia según el encargo", señaló el arquitecto a cargo del proyecto Piedra Blanca. Aún así, este proyecto se destacó por tener un nivel de desarrollo y detalle de la información BIM muy superior, incluyendo, por ejemplo, detalles y despiece de muebles de cocina. Cabe indicar que en este proyecto los profesionales de diseño participaron activamente durante la fase de construcción.

Los proyectos Casa Lican y Casa Las Colinas mostraron métodos de trabajo BIM no integrado multilateral. En el caso Lican se uso Vectorworks como plataforma base de trabajo, lo que representa una diferencia a la tendencia general de usar Revit y ArchiCAD. En ambos casos, los arquitectos utilizaron los modelos para realizar tareas y análisis más avanzados: estudios de sombra, radiación y confort térmico en Casa Licán, y de cubicación precisa de materiales y estimación de costos en Casa Las Colinas.

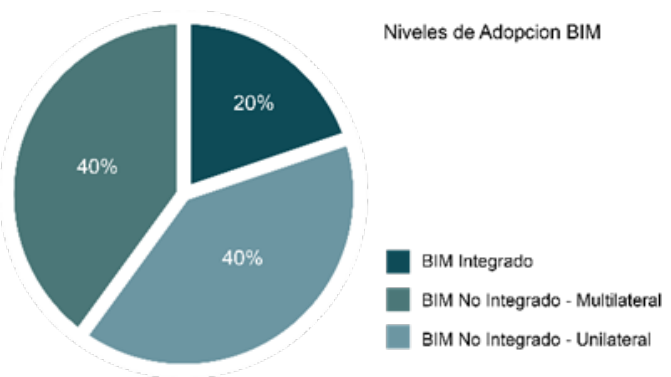

Figura 3. Modos de trabajo BIM en los casos.

En general, los beneficios observados y reportados son las mejoras en la disminución en errores en obra, mayor eficiencia en el uso de materiales y mejor calidad constructiva general, lo que es coincidente con lo reportado en la literatura para proyectos de mayor escala (Tabla 3).

Notablemente, otros beneficios que también aparecen destacados son la mejor comprensión del proyecto por parte de los trabajadores de terreno y la consecuente reducción de tiempo total de construcción debido a la menor cantidad de esperas por instrucciones. Esto marca una diferencia respecto a los proyectos de mayor escala, donde la organización de las obras es diferente y los trabajadores normalmente no tienen acceso a los modelos BIM ni a su información directamente. En obras de menor escala, la distancia entre obrero, 
supervisor y modelo BIM es más corta, y por tanto, la información fluye más fácilmente a todos los participantes.

En relación con las dificultades observadas (Tabla 4), sin duda el principal problema es la falta de profesionales especialistas que utilicen BIM. Esta limitación obliga a los profesionales que sí usan la tecnología a recurrir a formatos DWG para recibir y enviar información, lo que ralentiza todo el proceso e introduce riesgo de errores, omisiones y descordinaciones.

Tabla 3. Beneficios observados y reportados del uso BIM en pequeña escala

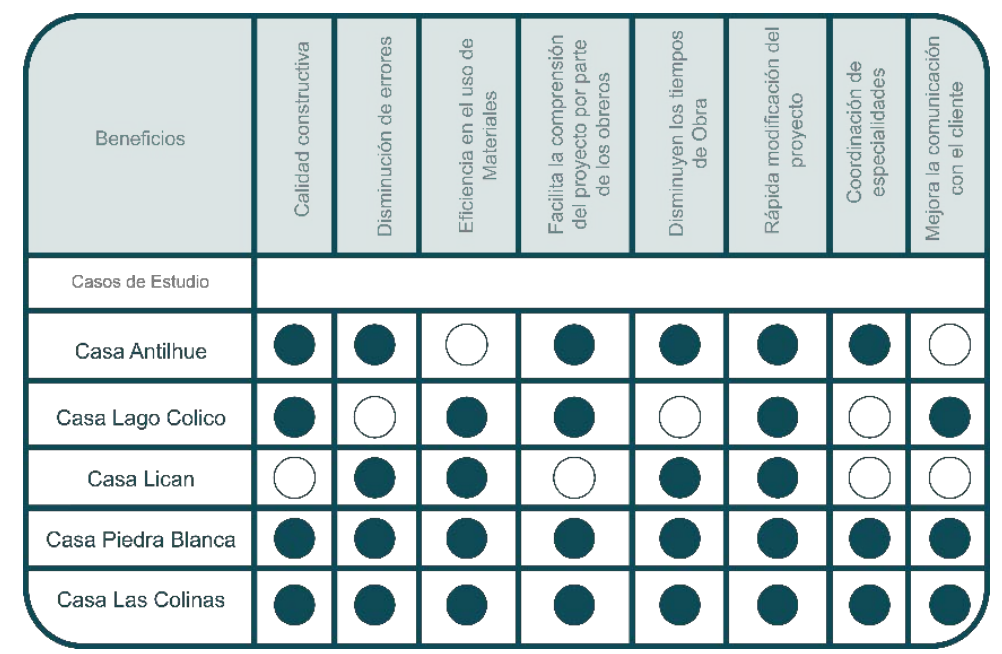

Tabla 4. Dificultades observadas y reportadoa del uso BIM en pequeña escala

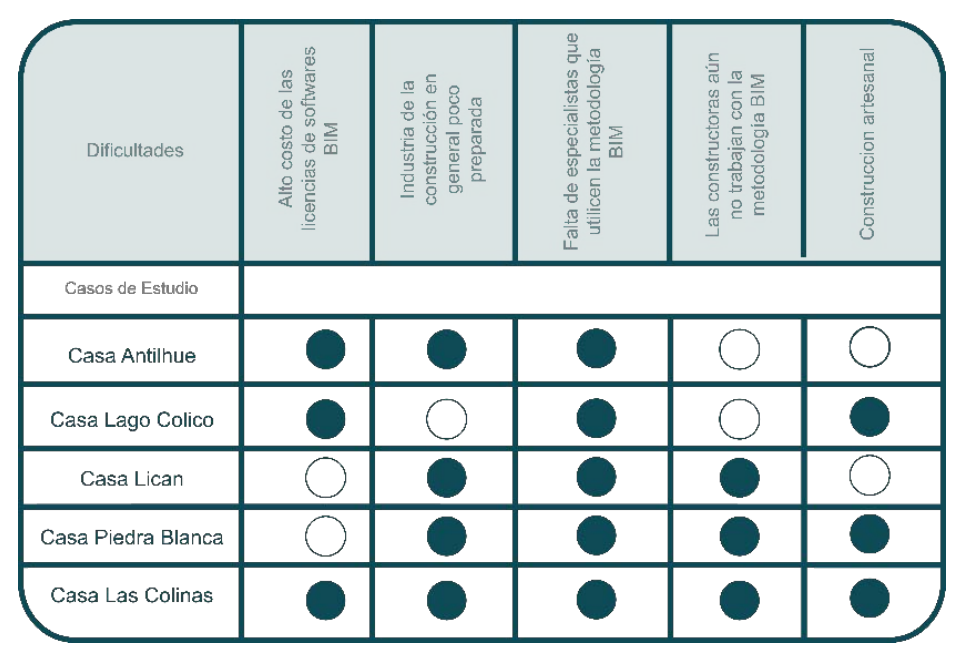




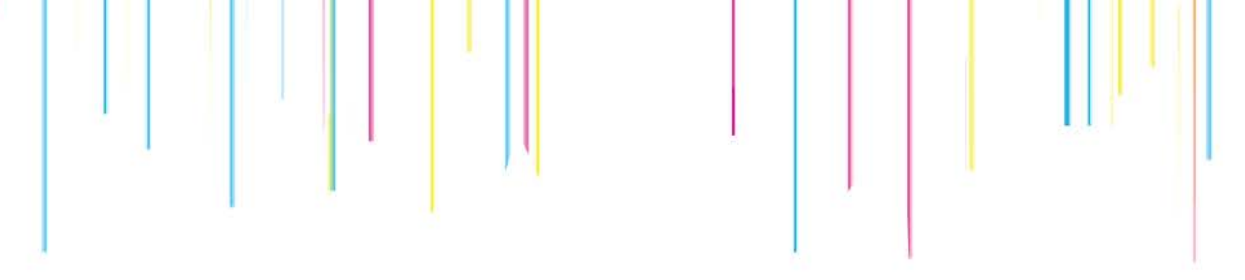

produce un subsidio natural entre proyectos de diferente envergadura, pero en oficinas orientadas exclusivamente a proyectos menores (como ampliaciones, remodelaciones) la barrera económica se vuelve infranqueable.

\section{CONCLUSIÓN}

Este trabajo realizó un análisis comparativo en profundidad sobre el impacto del uso de BIM en la mejora de la constructividad en proyectos de pequeña escala en Chile. En general, los resultados muestran que los beneficios obtenidos son similares a los reportados en la literatura para proyectos de mayor envergadura, por lo que se sugiere que no existen diferencias significativas en el uso de la tecnología para la mejora de la constructividad entre proyectos de pequeña o gran escala.

Agradecimientos. Agradecemos a los arquitectos Felipe Soffia, Benjamín Escabini, Francisco Javier Domínguez, Pablo Lobos y Claudio Labarca por su tiempo y por permitirnos estudiar sus proyectos; y a Ignacio Falcone, Juan Pablo Morales y Paulo Ogino por sus valiosos comentarios que ayudaron a encaminar este trabajo.

\section{$7 \quad$ Referencias}

Adams, S. (1990). Constructividad. Barcelona: Ediciones CEAC.

Alario, E. (2014). BIM a pequeña escala [Mensaje en un blog]. Recuperado de: https://enriquealario.com/bim-pequena-escala

BIM Forum Chile. (2017). Guía inicial para implementar BIM en las organizaciones. Santiago, Chile: BIM Forum Chile.

Bryde, D., Broquetas, M., Volm, J. (2013). The project benefits of Building Information Modelling (BIM), International Journal of Project Management, 31 (7), 971-980.

Camelio, N. (2011). La constructividad de edificios de geometrías complejas: las tecnologías digitales en el proceso de diseño y construcción. (Tesis de Pregrado). Universidad de Chile, Santiago, Chile.

Construction Industry Research and Information Association [CIRIA]. (1983). Buildability: An Assesment. Londres: CIRIA.

García, J. (2017). Metodología BIM en la realización de proyectos de construcción. Estudio de 6 viviendas adosadas en Gilet. (Tesis de Pregrado). Universidad politécnica de Valencia, Valencia, España. 
Guadalajara, C. (2017). Incidencia del BIM en el proceso proyecto-construcción de arquitectura (tesis de pregrado). Universidad Politécnica de Valencia, Valencia, España.

Klaschka, R. (2014). BIM in Small Practices: llustrated Case Studies. Newcastle, UK: NBS.

Loyola, M. y Goldsack, L. (2010). Constructividad y arquitectura: cómo mejorar la eficiencia de construcción desde el diseño. Santiago de Chile: LOM.

Tabilo, M. (2019). Estudio de la metodología BIM en la gestión de la construcción y aplicación demostrativa. (Tesis de pregrado). Universidad de Chile, Santiago, Chile.

Testa, R. (2019). Implementación BIM en la Dirección de Proyectos de Construcción. (Tesis de postgrado) Universidad de Valladolid, Valladolid, España.

Valdez, A. (2014). Estudio de viabilidad de uso de la tecnología BIM en un proyecto habitacional en altura. (Tesis de postgrado). Universidad de Chile, Santiago, Chile. 